\title{
Inpatient and outpatient loop electrosurgery excision procedure for cervical intraepithelial neoplasia: a retrospective analysis
}

\author{
B. Leimbacher $\cdot$ N. Samartzis $\cdot$ P. Imesch . \\ K. J. Dedes • D. Fink • C. Canonica
}

Received: 17 May 2011/ Accepted: 11 November 2011/Published online: 25 November 2011

(C) Springer-Verlag 2011

\begin{abstract}
Purpose To determine whether the outpatient loop electrosurgical excision procedure (LEEP) conization (outLEEP) is as effective and safe as inpatient LEEP conization (in-LEEP) with regard to the complete removal of cervical dysplasia, recurrence-free survival and post-operative morbidity.

Methods 233 patients were included in this retrospective cohort study from January 2002 to December 2007. 181 had outpatient treatment and 52 inpatient treatment. We used Mann-Whitney $U$ test, two-sided Fisher's exact test, Chi-square test, log rank test and Kaplan-Meier curve.

Results Incomplete excision was found in 16/52 (30.8\%) cases in the inpatient group and 46/181 (25.4\%) in the outpatient group $(P=0.48)$. Six patients had post-operative complications: two cases of secondary haemorrhage in each group (in-LEEP $3.8 \%$, out-LEEP $1.1 \%, P=0.22$ ) and two cases of cervical stenosis amongst inpatients (3.8\%, $P=0.049$ ). Alteration of specimen by thermal artifact were reported in 4/52 $(7.7 \%)$ of in-LEEP cones and $10 / 181(5.5 \%)$ of out-LEEP cones $(P=0.52)$. Measurements of cones in both groups were comparable with a mean depth of $9.35 \mathrm{~mm}( \pm 5.5 \mathrm{~mm})$ and $8.4 \mathrm{~mm}$ $( \pm 3.4 \mathrm{~mm})$, respectively.

Conclusion Our results suggest that efficacy and safety of ambulatory LEEP conization is comparable as in inpatient procedure.
\end{abstract}

B. Leimbacher · N. Samartzis · P. Imesch ·

K. J. Dedes $(\bowtie) \cdot$ D. Fink - C. Canonica

Department of Gynecology, University Hospital of Zurich,

Frauenklinikstrasse 10, 8091 Zurich, Switzerland

e-mail: konstantin.dedes@usz.ch
Keywords LEEP · Conization - Cervical intraepithelial neoplasia

\section{Introduction}

Loop electrosurgery excision procedure (LEEP) or, also referred as, large loop excision of the transformation zone (LLETZ) biopsy was introduced in 1989 by Prendiville et al. [1] as a novel technique to treat cervical intraepithelial neoplasia (CIN). LEEP conization offers the advantages of cold knife conization, i.e. the complete histological examination of the removed tissue and those of the local destructive procedures. The Cochrane collaboration database offers a review of the prospective randomized trials of different conization procedures [2]. Compared to cold knife and laser conization, LEEP has equal efficacy and safety. The frequency of residual disease, intra and post-operative haemorrhage, cervical stenosis and secondary sterility is not significantly higher amongst LEEP treated patients [2, 3].

Since LEEP conization can be performed under local anaesthesia in an ambulatory setting, hospitalisation and general anaesthesia can be avoided [4, 5], and LEEP conization has gained wide acceptance [6, 7].

The purpose of this study was to examine whether outpatient LEEP conization is as effective as inpatient LEEP conization with regard to complete removal of cervical dysplasia and recurrence-free survival (RFS), to assess the safety of outpatient treatment and frequency of post-operative complications.

Literature never examined the efficacy and safety of LEEP comparing inpatient and outpatient procedures. It is unclear if these two approaches of the same surgical technique achieve a similar satisfactory result concerning the complete removal of cervical intraepithelial neoplasia. 


\section{Materials and methods}

We retrospectively reviewed the medical records of patients who underwent LEEP conization for the treatment of cervical intraepithelial neoplasia (CIN) in the Department of Gynecology of the University Hospital of Zurich from January 2002 to December 2007.

Requirement of patients and fear of local anaesthesia are the reasons to perform LEEP as an inpatient instead of an outpatient procedure.

The indications for conization were (1) biopsy proven CIN2-3, (2) persistent CIN1, or (3) discrepancy between cytology, biopsy, and/or colposcopy in women with Papanicolaou smears showing high-grade squamous intraepithelial lesions (HSIL). Patients were only included if they never had a conization before.

Gynaecologists in both settings were equally skilled and had at least a consultant degree. In the outpatient setting, surgery was performed under local anaesthesia. A cervical block was achieved by the injection of $1 \%$ lidocain into the cervix. Inpatient LEEP was performed under general or regional anaesthesia, depending on the feasibility and the patient preference.

Before conization, all patients had a colposcopic examination with the application of acetic acid followed by Lugol iodine solution for the localization of cervical lesions. LEEP was performed with an electrosurgical unit (ERBE, Swiss AG) using $100 \mathrm{~W}$. For cervical conization, we used tungsten wire loop electrodes (Valleylab, Boulder, Colorado, USA) with loop size of $10 \times 10 \mathrm{~mm}$ (E1559), $15 \times 12 \mathrm{~mm}($ E1560) or $20 \times 12 \mathrm{~mm}$ (E1561), depending on the lesion extent. In the majority of cases specimens were removed in one loop pass, if required a second pass was done during the same intervention. Haemostasis was achieved by LLETZ Ball Electrode (E 1564) set to $60 \mathrm{~W}$ in spray mode. Post-excisional Lugol solution was applied to confirm macroscopic free margins.

Specimens were marked by a single suture at 12 o'clock for orientation, and fixed in formalin. The depth of the cone and the largest diameter at its base were measured. These measurements were compared between the two settings. Margins were inked for identification. The cones were serially sectioned and entirely embedded. We defined two groups of surgical margins, those with negative and those with non-negative margins. The non-negative group comprises cases with positive margins as well as equivocal cases due to the thermal artifact.

Post-operative haemorrhage was assessed and compared in the two groups. Follow-up cytologic testing and colposcopy were scheduled between 4 and 6 months after conization, and repeated every 6 months until two normal Papanicolaou smears were obtained. Cervical biopsy and endocervical curettage were performed as indicated for an abnormal cytologic result or colposcopic lesion. Cervical stenosis was diagnosed, if introduction of a Hegar dilator $3 \mathrm{~mm}$ failed.

Potential risk factors for developing CIN or recurrence have been suggested to include smoking [8], oral contraceptives (OC) [8], HIV [9], immunosuppressive therapy (IST) $[10,11]$ and high parity [8]. These factors were recorded for both groups.

Mann-Whitney $U$ test was used to compare continuous data (age, follow-up time, cone measurements) between the two intervention groups. Two-sided Fisher's exact test and Chi-square test were applied to evaluate categorical data, such as risk factors, histological diagnosis, margin involvement and cautery artifact. The post-operative RFS was compared by log rank test. Patients with hysterectomy or repeated conization or a follow-up less than 6 months were excluded from the log rank test and Kaplan-Meier curve. $P$ values lesser than 0.05 were considered significant. Statistical analyses were performed using SPSS 18.0 package software (SPSS Inc., Chicago, IL, USA).

\section{Results}

Of the 233 patients included in our study, 52 (22\%) underwent an inpatient and 181 (78\%) an outpatient treatment. Thirteen patients were censored because pathological investigation was not possible or did not show CIN in the colposcope directed punch biopsy or conization specimen. Table 1 summarizes the clinical data of patients in both groups. Mean age was comparable in both groups. Mean follow-up time in the inpatient group was 19.5 (0-61) months and $14.8(0-58)$ months for outpatient group. The severity of cervical dysplasia in both cohorts was similar. There were three cases of previously unsuspected invasive cancer in conization specimens. While no significant difference in frequency of HIV seropositivity was found, the prevalence of tobacco smoking and OC were not equal in both groups. To assess the impact of this heterogeneity, we analyzed the association of these variables with the surgical margin and RFS. In the grouped analyses (Fisher's exact test), no significant associations between specimen margins and tobacco smoking or OC use were observed $(P=0.28, P=0.50) .10 / 50(20 \%)$ smokers and 52/183 (28\%) of non-smokers had positive margins, and similar numbers of patients with and without OC treatment were $7 / 33(21 \%)$ and 30/104 (29\%). The odds ratio for incomplete resection among tobacco smoking was 0.63 [95\% confidence interval (CI), 0.29-1.35] and amongst OC treatment 0.66 (95\% CI, 0.26-1.69). No significant differences in log rank analysis of RFS for tobacco smoking or $\mathrm{OC}$ use were found $(P=0.72$ and $P=0.21$, respectively). 
Table 1 Patient characteristics and final diagnosis

\begin{tabular}{lccc}
\hline & Inpatient LEEP & Outpatient LEEP & $P$ value \\
\hline Age (years) & $33.3( \pm 9.2)$ & $34.5( \pm 8.3)$ & $0.31^{\circ}$ \\
Follow-up (months) & $19.5( \pm 16.6)$ & $14.8( \pm 14.8)$ & $0.08^{\circ}$ \\
Histological diagnosis & & & \\
CIN 1 & $13 / 52(25.0 \%)$ & $43 / 181(23.8 \%)$ & $0.90^{* *}$ \\
CIN 2 & $12 / 52(23.1 \%)$ & $41 / 181(22.7 \%)$ & \\
CIN 3 & $26 / 52(50.0 \%)$ & $95 / 181(52.5 \%)$ & \\
Invasive cancer & $1 / 52(1.9 \%)$ & $2 / 181(1.1 \%)$ & \\
Risk factors & & & \\
HIV & $4 / 52(7.7 \%)$ & $6 / 181(3.3 \%)$ & $0.24^{*}$ \\
Tobacco & $18 / 52(34.6 \%)$ & $32 / 181(17.7 \%)$ & $\mathbf{0 . 0 1 2}$ \\
OC & $7 / 42(14.3 \%)$ & $26 / 88(29.5 \%)$ & $\mathbf{0 . 0 3 4}$ \\
Parity & $0.5( \pm 1.0)$ & $0.7( \pm 0.9)$ & $0.23^{\circ}$ \\
IST & $1 / 52(1.9 \%)$ & $6 / 181(3.3 \%)$ & $1.00^{*}$ \\
\hline Age, folow-up & & &
\end{tabular}

Age, follow-up and parity are given with mean and standard deviation in brackets. Oral contraceptives (OC) was reported only for 42 and 88 patients in the in-LEEP and out-LEEP group, respectively; records of parity missed in 49 and 73 patients of the in-LEEP and out-LEEP setting, respectively. Bold values are statistically significant $(P<0.05)$

$O C$ oral contraceptives, $I S T$ immunosuppressive therapy

$P$ values were estimated by ${ }^{\circ}$ Mann-Whitney $U$ test, *Fisher's exact test or $* *$ Chi-square test

Depth of specimen was not assessed in 22 cases in the ambulatory setting (12\%). The mean depth of cone specimen in the inpatient setting was $9.35 \mathrm{~mm}$ (standard deviation, SD 5.5) and $8.4 \mathrm{~mm}$ (SD 3.4) for outpatient treatment (Fig. 1a). The mean diameter of the base of the cone was $12.8 \mathrm{~mm}$ (SD 7.0) and $13.5 \mathrm{~mm}$ (SD 6.8) for inand outpatient, respectively. No statistical significant difference was found of depth $(P=0.71)$ and diameter $(P=0.16)$ between the groups.
Table 2 Prevalence of non-negative surgical margins in the two cohorts

\begin{tabular}{lrcc}
\hline & Inpatient LEEP & Outpatient LEEP & $P$ value \\
\hline Total & $16 / 52(30.8 \%)$ & $46 / 181(25.4 \%)$ & 0.48 \\
Endocervical margin & $14 / 52(26.9 \%)$ & $33 / 181(18.2 \%)$ & 0.17 \\
Ectocervical margin & $9 / 52(17.3 \%)$ & $23 / 181(12.7 \%)$ & 0.37 \\
CIN & & & \\
1 & $2 / 13(15.4 \%)$ & $4 / 43(9.3 \%)$ & 0.62 \\
$2-3$ & $13 / 38(34.2 \%)$ & $41 / 136(30.1 \%)$ & 0.69 \\
Age (years) & & & \\
$\leq 40$ & $12 / 39(30.8 \%)$ & $33 / 141(23.4 \%)$ & 0.40 \\
$>40$ & $4 / 13(30.8 \%)$ & $13 / 40(32.5 \%)$ & 1.00 \\
Parity & & & \\
$<1$ & $8 / 33(24.2 \%)$ & $8 / 42(19.0 \%)$ & 0.78 \\
$\leq 1$ & $7 / 16(43.8 \%)$ & $7 / 31(22.6 \%)$ & 0.18 \\
Tobacco smoking & & & \\
No & $11 / 34(32.4 \%)$ & $41 / 149(27.5 \%)$ & 0.67 \\
Yes & $5 / 18(27.8 \%)$ & $5 / 32(15.6 \%)$ & 0.46 \\
Oral contraceptives & & & \\
No & $13 / 42(31.0 \%)$ & $17 / 62(27.4 \%)$ & 0.83 \\
Yes & $2 / 7(28.6 \%)$ & $5 / 26(19.2 \%)$ & 0.62 \\
\hline All $P$ vals & &
\end{tabular}

All $P$ values results from Fisher's exact test

The incomplete excision rate was $30.8 \%$ in the inpatient group and $25.4 \%$ in the outpatient group. These proportions were not significantly different, even following separate analysis of endo- and ectocervical margin (Table 2).

Table 3 shows frequency of cautery artifact and operative outcome in both groups. Two cases of cervical stenosis were identified in the inpatient treatment. Their specimen size were $16 \times 15$ and $11 \times 18 \mathrm{~mm}$ (depth $\times$ diameter). Conus depths were higher than in cones from complication-free patients. But correlation of cervical stenosis with
Fig. 1 a Depth of conization specimen. Extreme values are represented by circles.

b Kaplan-Meier curve of inpatient (continuous line) and outpatient group (dashed line)
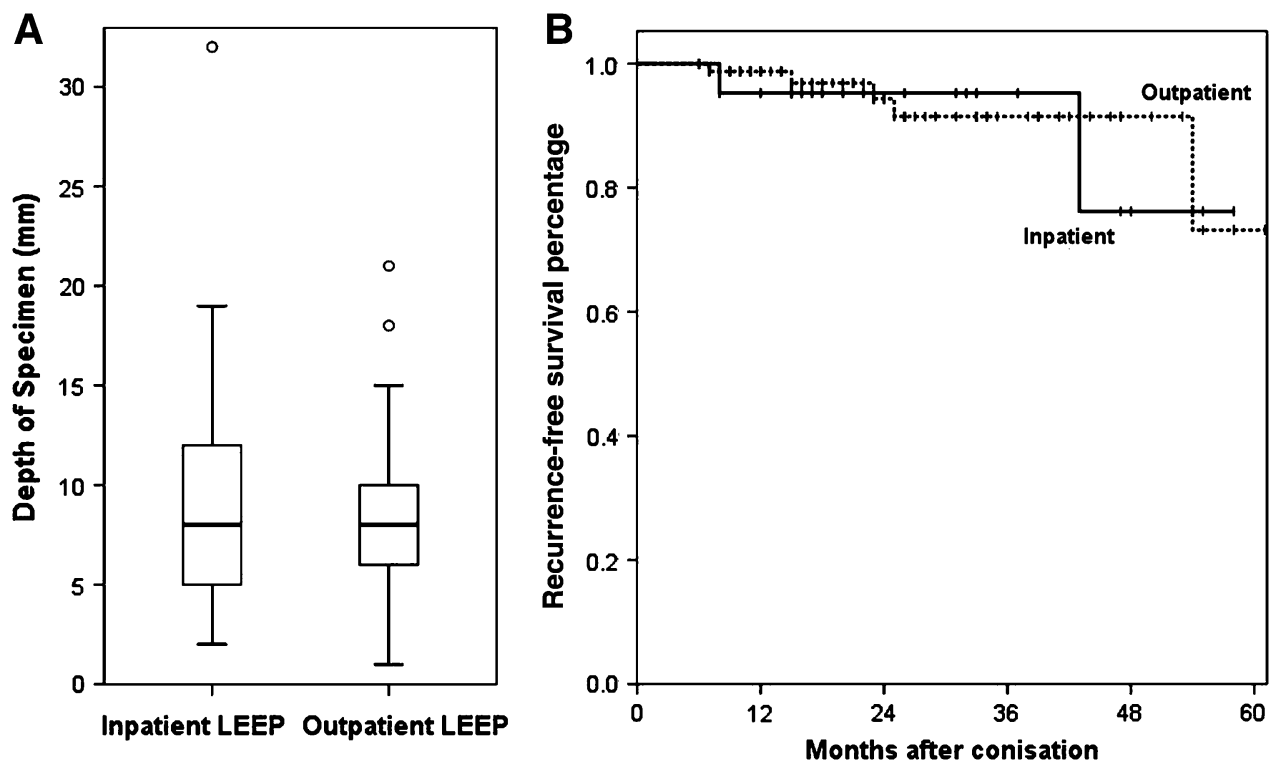
Table 3 Outcome in the two treatment groups

\begin{tabular}{llll}
\hline & $\begin{array}{l}\text { Inpatient } \\
\text { LEEP }\end{array}$ & $\begin{array}{l}\text { Outpatient } \\
\text { LEEP }\end{array}$ & $P$ value \\
\hline $\begin{array}{l}\text { Thermal artifact on } \\
\text { specimen }\end{array}$ & $4 / 52(7.7 \%)$ & $10 / 181(5.5 \%)$ & $0.52 *$ \\
$\begin{array}{l}\text { Complication } \\
\text { Total }\end{array}$ & & & \\
Haemorrhage & $4 / 52(7.7 \%)$ & $2 / 181(1.1 \%)$ & $\mathbf{0 . 0 2 4} *$ \\
Cervical stenosis & $2 / 52(3.8 \%)$ & $2 / 181(1.1 \%)$ & $0.22 *$ \\
Infection & $2 / 52(3.8 \%)$ & $0 / 181(0 \%)$ & $\mathbf{0 . 0 4 9}$ \\
\hline
\end{tabular}

All $P$ values results from Fisher's exact test. Bold values are statistically significant $(P<0.05)$

depth or diameter were not significant $(P=0.07$, $P=0.13$, Mann-Whitney $U$ test). RFS curves of the inpatient and outpatient collective are shown on Fig. 1b. Univariate log rank analysis did not show a significant difference $(P=0.81)$.

\section{Discussion}

The aim of this study was to determine whether the outpatient LEEP conization is comparable to the inpatient LEEP conization. Efficacy was not significantly different in both cohorts, with a total of $74.6 \%$ dysplasia-free margin in the outpatient group and $69.2 \%$ in the inpatient group. RFS did not significantly differ between both groups.

Both group's age and severity of cervical dysplasia were comparable. Only the frequency of smoking and the use of OC differed in terms of the two cohorts. The impact of this appears limited between odds ratios, as odds ratios of these properties to the involvement of surgical margin were both close to one, confidence interval of $95 \%$ crossed the level one mark and did not have a major difference to recurrence-free time. In addition, outpatient and inpatient setting subdivided into smoker and non-smoker, as well as hormones intake and hormone-free population to ensure an uninfluenced cohort analysis, showed similar $P$ values.

Safety of LEEP conization appeared comparable. Outpatient treatment was not inferior to the inpatient treatment in terms of secondary haemorrhage, cervical stenosis or post-operative infections. In fact, cervical stenosis was observed even less frequently in the outpatient group compared to the inpatient group. Related to the literature, the frequency of clear margin excisions, secondary haemorrhages and cervical stenoses in our study were comparable to those reported by other groups $[12,13]$.

Sadler et al. [14] reported that LEEP conization can lead to a fragility of cervical connective tissue during pregnancy which may predispose patients to preterm birth. For cone depths over $17 \mathrm{~mm}$, there was a significant risk of preterm delivery. In the present study, the depth of the excised cone in the outpatient group was not significantly higher.

Ambulatory LEEP treatment may provide lower costs than hospitalization and general anaesthesia. However, there is no such evaluation of cost-effectiveness in the literature, with regard to hysteroscopy, which also can be performed as an in- and outpatient procedure, a British study estimated $50 \%$ reductions in cost for outpatient management compared to short hospital stay [15].

In conclusion, LEEP conization can be performed under local anaesthesia in an ambulatory setting, avoiding hospitalization and general anaesthesia. It represents a feasible option for the management of cervical dysplasia. Efficacy and safety are comparable to inpatient management.

Conflict of interest All authors declared there is no conflicts of interest related to this work.

\section{References}

1. Prendiville W, Cullimore J, Norman S (1989) Large loop excision of the transformation zone (LLETZ). A new method of management for women with cervical intraepithelial neoplasia. Br J Obstet Gynaecol 96(9):1054-1060

2. Martin-Hirsch PP, Paraskevaidis E, Bryant A, Dickinson HO, Keep SL. Surgery for cervical intraepithelial neoplasia. Cochrane Database Syst Rev. (6):CD001318

3. Mathevet P, Chemali E, Roy M, Dargent D (2003) Long-term outcome of a randomized study comparing three techniques of conization: cold knife, laser, and LEEP. Eur J Obstet Gynecol Reprod Biol 106(2):214-218

4. Mor-Yosef S, Lopes A, Pearson S, Monaghan JM (1990) Loop diathermy cone biopsy. Obstet Gynecol 75(5):884-886

5. Fanning J, Padratzik J (2002) Cold knife conization vs. LEEP. Are they the same procedure? J Reprod Med 47(1):33-35

6. Tyler LN, Andrews N, Parrish RS, Hazlett LJ, Korourian S (2007) Significance of margin and extent of dysplasia in loop electrosurgery excision procedure biopsies performed for highgrade squamous intraepithelial lesion in predicting persistent disease. Arch Pathol Lab Med 131(4):622-624

7. Wright TC Jr, Gagnon S, Richart RM, Ferenczy A (1992) Treatment of cervical intraepithelial neoplasia using the loop electrosurgical excision procedure. Obstet Gynecol 79(2): $173-178$

8. Castellsague X, Munoz N (2003) Chapter 3: cofactors in human papillomavirus carcinogenesis-role of parity, oral contraceptives, and tobacco smoking. J Natl Cancer Inst Monogr 31:20-28

9. Tebeu PM, Major AL, Mhawech P, Rapiti E (2006) The recurrence of cervical intraepithelial neoplasia in HIV-positive women: a review of the literature. Int J STD AIDS 17(8):507-511

10. David M, Olbrich C, Neuhaus R, Lichtenegger W (1995) Occurrence of suspicious changes in cervix cytology in women after liver transplantation. Geburtshilfe Frauenheilkd 55(8):431-434

11. Gitsch G, Kainz C, Pohanka E, Reinthaller A, Kovarik J, Tatra G et al (1992) Human papillomavirus infection of the uterine cervix in immune suppressed women after kidney transplantation. Geburtshilfe Frauenheilkd 52(12):764-766 
12. Vejerslev LO, Schouenborg L, Sorensen F, Nielsen D, Sorensen SS, Juhl BR et al (1999) Loop diathermy or laser excisional conization for cervical intraepithelial neoplasia. Acta Obstet Gynecol Scand 78(10):900-905

13. Duggan BD, Felix JC, Muderspach LI, Gebhardt JA, Groshen S, Morrow CP et al (1999) Cold-knife conization versus conization by the loop electrosurgical excision procedure: a randomized, prospective study. Am J Obstet Gynecol 180(2 Pt 1):276-282
14. Sadler L, Saftlas A, Wang W, Exeter M, Whittaker J, McCowan L (2004) Treatment for cervical intraepithelial neoplasia and risk of preterm delivery. JAMA 291(17):2100-2106

15. Marsh F, Kremer C, Duffy S (2004) Delivering an effective outpatient service in gynaecology. A randomised controlled trial analysing the cost of outpatient versus daycase hysteroscopy. BJOG 111(3):243-248 